\title{
Break-Even Point for a Proof Slip Operation
}

\begin{abstract}
This article applies break-even analysis to determine what magnitude of titles added per year (for which a proof slip will be used in producing card sets) is sufficient to utilize economically Library of Congress proof slips and a Xerox 914 copying machine in the cataloging operation of a library. A break-even formula is derived, and an example of its use is given using data gathered at Arkansas State University.
\end{abstract}

$T$ HIS ARTICLE aims to derive a formula which will determine the economic break-even point for the use of Library of Congress proof slips and a Xerox 914 copying machine in the cataloging operation of a library. An example of the use of this formula will be given using statistics gathered from the cataloging department of the Arkansas State University library.

As the numerous uses of LC proof slips in libraries have been elucidated in an article authored by Samuel Waters and Salvatore Costabile, this article will focus upon the uses of proof slips only as they pertain to the production of catalog cards. ${ }^{1}$ Derivation of the formula will not reflect the fact that most libraries using a Xerox 914 to reproduce catalog cards defray their rental costs through much more extensive use of the

1 Samuel T. Waters and Salvatore L. Costabile, "The Proof of the Pudding: Using Library of Congress Proof Slips," CRL 27:87-91 (March 1967).

Mr. Anderson was head cataloger, Arkansas State University library at the time this article was written. He is now director, First Regional Library, Hernando, Mississippi. machine than through mere reproduction of catalog cards from proof slips; on the contrary, it will be assumed that the reproduction of catalog cards from proof slips must defray the entire cost of the Xerox 914. This assumption will, of course, necessitate a very conservative break-even point figure, and any library using the formula to determine whether or not to use proof slips should bear this in mind. One other advantage of proof slips which this formula does not account for is the time savings accrued. The economic advantage of having immediate access to proof slips over delaying cataloging while cards are ordered from the Library of Congress is very difficult to assess quantitatively, and is therefore not included in this analysis.

There are three constants and two variables which comprise this equation. The constants are defined as the cost of LC card sets, the cost of Xeroxing a complete set of cards, and the cost of a proof slip subscription. The variables are the cost of student filing per year and the number of titles ordered per year. While the latter is clearly a variable dependent upon the individual library, the student filing cost varies only 
as the types of arrangement, types of titles needed, and depth of proof slip file vary.

The break-even point is derived when the cost of using proof slips in the cataloging process equals the cost of ordering Library of Congress cards for each title. Represented symbolically, this is defined as

$$
a x=b x+c+d
$$

where:

$a=$ cost of LC cards/set,

$\mathrm{b}=$ Xerox cost for one set of cards,

$\mathrm{c}=$ cost of proof slip subscription/year,

$\mathrm{d}=$ cost of student filing/year, and

$\mathrm{x}=$ number of titles added per year.

When (bx) is subtracted from both sides of the equation, the formula becomes:

$$
\begin{aligned}
a x-b x & =c+d, \text { or } \\
x(a-b) & =c+d
\end{aligned}
$$

The variable $(x)$ can then be isolated by dividing both sides of the equation by $(a-b)$ :

$$
x=\frac{c+d}{a-b}
$$

Therefore, when the number of titles which would have a proof slip available is greater than the quantity $\frac{c+d}{a-b}$, it would be more economical to use proof slips rather than order LC card sets for each title received.

In order to suggest how this formula might be applied in a library, and to furnish statistics which other libraries might use in computing their fixed and variable costs for a proof slip operation of this type, an actual example is presented in the following section. It is also hoped that this example will help clarify the usefulness of cost figures.

When Arkansas State University decided in 1967 to begin a subscription to Library of Congress proof slips, it was agreed that the basic need, as far as cataloging was.concerned, was for current imprints in the English language. Accordingly, the procedure for handling proof slips was geared toward maintaining a three-year file of proof slips of titles in the English language. This meant that upon receiving the weekly box of proof slips from the Library of Congress, all slips for books with a copyright date older than three years, as well as those for foreign language books, were discarded. The remaining slips were alphabetized by main entry and filed in the appropriate section of the catalog for the particular year in which the book was copyrighted. At the beginning of each new year, the oldest section was discarded, thus maintaining the three-year file. We found that a three-year file of English language proof slips could easily be housed in two sixty-tray catalogs.

Over a one-year period, the number of hours spent each day sorting, alphabetizing, and filing proof slips were totaled by the filers in the cataloging department. These statistics totaled approximately 485 hours. This figure, multiplied by the hourly wage rate for student workers, $\$ 1.60$, yielded the figure $\$ 776$, and accounts for variable " $\mathrm{d}$ " in the equation. The other fixed cost is quantity " $\mathrm{c}$ " in the formula, which is the yearly cost of a subscription to the proof slips. This cost, which is subject to change every year, was $\$ 385$ last year.

The two variable costs (costs that vary according to the number of books cataloged) are the cost of LC card sets and the cost of Xeroxing a card set from a proof slip. The cost of an LC card set was $\$ .35$. The cost of Xeroxing a card set was obtained by adding the average rental cost per card of a Xerox $914(\$ .01)$ to the average cost per card of card stock (\$.005), yielding a total of $\$ .015$ per card. The operator time for the Xerox 914 is not figured into this to$\mathrm{tal}$, because it is assumed that this time is offset by the time saved by typing the call number on the proof slip to be 
Xeroxed, thereby eliminating the necessity of typing the call number on each card in the complete set. Multiplying this figure by five, which is the average number of cards per set, we produced quantity " $\mathrm{b}$ " in the equation, a figure of $\$ .075$.

When these figures are substituted into the original equation:

$$
\begin{aligned}
& x=\frac{385+776}{0.35-0.075} \text { or } \\
& x=4222
\end{aligned}
$$

The reader should bear in mind that this figure is not the total number of titles which must be added each year in order to realize the economies of a proof slip subscription, but rather the total number of titles for which a proof slip must be used to produce card sets.

It is the opinion of the author that a break-even analysis such as the one described in this article can be a very useful tool to librarians. Aside from the many other uses of proof slips, a medium-sized library can realize a definite savings in processing costs by utilizing a proof slip subscription and a Xerox 914 copier instead of ordering card sets from the Library of Congress. 